
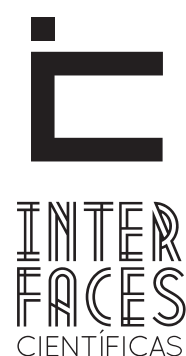

HUMANAS E SOCIAIS

ISSN IMPRESSO 2316-3348

ISSN ELETRÔNICO 2316-3801

DOI 10.17564/2316-3801.2015v3n3p113-122

\title{
A ENERGIA COMO UM REVERSO SALIENTE NO SISTEMA SOCIOTÉCNICO DO BRASIL
}

Delano Mendes de Santana

Douglas Alves Cassiano ${ }^{3}$
Sérgio Ricardo Lourenço ${ }^{2}$

\section{RESUMO}

A abordagem do sistema energético nacional como um sistema sociotécnico é fundamental para fomentar o diálogo dessa questão com a sociedade. A partir de uma metodologia do tipo exploratória, foi construída uma hipótese sob a ótica do conceito de reverso saliente proposto por Thomas Hughes. Foram apresentadas relações complexas de interdependência entre aspectos físicos e técnicos, sociais, ambientais, econômicos e políticos, onde a cada mudança de padrão de elementos todos os demais precisam se adaptar para buscar o equilíbrio do sistema. Foi possível identificar pontos de aderência entre os conceitos propostos por Hughes e o sistema energético nacional. A partir da análise da tendência de crescimento da demanda de energia, a mudança nos padrões de consumo da sociedade e a estagnação da distribuição das fontes de energia nacionais, evidencia-se a produção de energia como um reverso saliente no contexto sociotécnico brasileiro e alerta-se para as necessidades de mudanças estruturais para reversão deste gargalo nacional.

\section{PALAVRAS-CHAVE}

Thomas Hughes. Energia. Sistemas Sociotécnicos. Reversos Salientes. 


\section{ABSTRACT}

The approach of the national energy system as a socio-technical system is critical to fostering this question dialogue with society. From a methodology of exploratory, was built a hypothesis from the perspective of the concept of reverse salient proposed by Thomas Hughes. Complex interrelationship between physical and technical, social, environmental, economic and political were presented, where each pattern change from all the other elements need to adapt to find the balance of the system. It was possible to identify points of adhesion between the concepts proposed by Hughes and the national energy system. From the analysis of energy demand growth trend, the change in society's consumption patterns and the stagnation of distribution of domestic energy sources, energy production is evident as a feature reverse in the Brazilian socio-technical context and alert up for the purposes of structural changes to reverse this national bottleneck.

\section{KEYWORDS}

Thomas Hughes. Energy. Socio-technical Systems. Salient Reverse.

\section{RESUMEN}

El enfoque del sistema energético nacional como un sistema socio-técnico es fundamental para mejorar este diálogo con la sociedad. A partir de una metodología de exploración, una hipótesis se construyó desde la perspectiva de los conceptos de Thomas Hughes. Compleja interrelación entre la física y técnica, social, ambiental, económica y política se presentaron, en la que cada cambio en el patrón todos los demás elementos tiene que adaptarse para encontrar el equilibrio del sistema. Fue posible identificar puntos de adhesión entre los conceptos y el sistema energético. A partir del análisis de la tendencia de crecimiento de la demanda de energía, el cambio en los patrones de consumo de la sociedad y el estancamiento de la distribución de las fuentes nacionales de energía, la producción de energía es evidente como reverso sobresaliente en el contexto socio-técnico brasileño y alerta a la los cambios estructurales necesarios para revertir este cuello de botella nacional.

\section{PALABRAS CLAVE}

Thomas Hughes. Energía. Sistemas Socio-técnicos. Reversos Sobresalientes. 


\section{INTRODUCÇÃO}

Thomas Parke Hughes (13 de setembro de 1923 - 3 de fevereiro de 2014) foi um historiador da tecnologia Americano. Emérito professor da Universidade da Pensilvânia, foi professor convidado do Massachusetts Institute of Technology (MIT) e da Universidade de Stanford. Os seus principais trabalhos acadêmicos tratam da evolução de grandes sistemas tecnológicos e as suas relações com a sociedade.

Especialista em sistemas elétricos elaborou uma abordagem abrangente que pode ser aplicada a grandes blocos tecnológicos integrados com a sociedade, denominados de sistemas sociotécnicos. Tais sistemas podem ser compostos por elementos técnicos como: turbo geradores, transformadores e linhas de transmissão, por exemplo. Podem incluir, também, as organizações públicas e privadas como as unidades fabris, empresas de serviços e bancos; ou incorporar componentes científicos como livros, artigos, professores universitários e programas de pesquisa. Hughes propõe que neste contexto abrangente, questões legislativas políticas como leis e regulamentos, também, fazem parte do sistema tecnológico.

O tratamento desta entidade complexa com aspectos técnico-científicos, econômicos, políticos e ambientais como uma construção social implica que não há nada epistemologicamente especial sobre a natureza deste conhecimento, sendo mais um elemento não diferenciado no conjunto de conhecimentos culturais humanos (BIJKER, 1984).

A Figura 1 apresenta a ilustração dos aspectos que envolvem um sistema sociotécnico em todas as suas esferas de abrangência.

\section{Figura 1 - Sistema sociotécnico}

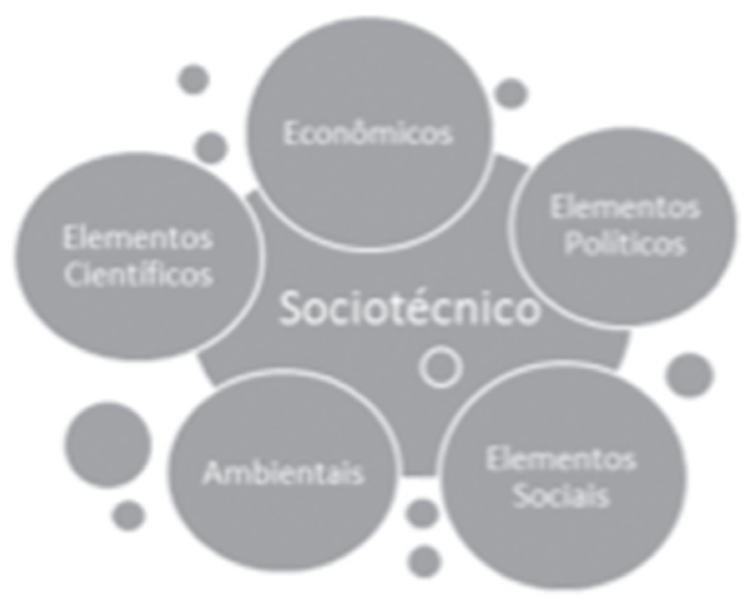

Fonte: Produção do próprio autor.

Não só o conhecimento, como cada um dos elementos, funcionando como componente chave do sistema tecnológico interage com os outros artefatos e contribui direta ou indiretamente com o objetivo comum do sistema. Se um componente é removido ou alterado no sistema, os demais necessitam se ajustar a esta mudança (HUGHES, BIJKER, PINCH, 1989).

O sistema energético nacional em questão foi abordado como um sistema sociotécnico. Como tal, segue o fluxo da sociedade humana e acompanha o crescimento da população brasileira.

Quando estes sistemas sociotécnicos crescem, outros tipos de problemas são desenvolvidos, alguns dos quais podem ser chamados de reversos salientes. Estes são elementos do sistema que ficaram obsoletos ou que estão fora de fase com os demais componentes. Sugerem mudanças complexas e se assemelham ao conceito de sistemas visualmente rígidos conhecidos como gargalos ou barreiras (HUGHES, BIJKER, PINCH, 1989). 
A abordagem da energia como um sistema sociotécnico é fundamental para fomentar o diálogo dessa questão no contexto social brasileiro. Como exemplo, pode-se citar a usina hidrelétrica de Santo Antônio em Porto Velho-RO. Esta usina tem previsão de conclusão em novembro de 2016 para operar com 50 turbinas de potência total de 3.568 MW.

Nesse contexto alguns impactos sociais precisam ser considerados. A barragem inundou uma área de $350 \mathrm{~km}^{2}$ e deslocou pelo menos 1.762 pessoas, sendo que grande parte são consideradas ribeirinhas, ou seja, que retira do rio sua principal fonte de subsistência, o peixe.Como mitigação destes e de outros danos socioambientais foi desenvolvido o Projeto Básico Ambiental (PBA) que em 2008 previa uma verba de $\mathrm{R} \$ 1,3$ bilhão em investimentos locais. Porém, por falhas no projeto e irregularidades na licitação, apontadas pela Controladoria Geral e pelo Tribunal de Contas, as obras foram paralisadas em 2010. Em 2014 a população local ainda sofria com problemas ocasionados pela falta de serviços básicos, estando o percentual de saneamento em torno de $2 \%$ (RIBEIRO e MORET, 2014).

Uma série de órgãos governamentais e privados, que serão descritos a seguir, dão suporte às informações relacionadas ao tema de energia e que serão fundamentais para a análise proposta neste trabalho.

No âmbito nacional, a Empresa de Pesquisa Energética (EPE) disponibiliza anualmente o Balanço Energético Nacional (BEN), fundamental para atividades de planejamento e acompanhamento do setor energético brasileiro. Contém a contabilidade da oferta e consumo de energia no Brasil e reúne em um único documento as séries históricas dessas operações, além das informações sobre reservas, capacidades instaladas e importantes dados estaduais (EPE, 2014).

No contexto da energia elétrica, a Agência Nacional de Energia Elétrica (ANEEL), autarquia em regime especial vinculada ao Ministério de Minas e Energia, tem por finalidade regular e fiscalizar a geração, transmissão, distribuição e comercialização de energia elétrica, segundo a legislação e as diretrizes do Governo Federal (ANEEL, 2013).

Já a Eletrobras é uma empresa de capital aberto, controlada pelo governo brasileiro, que atua nas áreas de geração, transmissão e distribuição de energia elétrica. Com foco em rentabilidade, competitividade, integração e sustentabilidade, a companhia lidera um sistema composto de 12 subsidiárias, uma empresa de participações (Eletrobras Eletropar), um centro de pesquisas (Eletrobras Cepel) e metade do capital da Itaipu Binacional (ELETROBRAS, 2014a).

A Confederação Nacional da Indústria (CNI) e a Eletrobras, por meio do - Programa de Conservação de Energia Elétrica (PROCEL) Indústria, desenvolvem estreita cooperação, desde 2004, com o objetivo de aumentar o dinamismo das ações de eficiência energética no setor industrial (CNI e ELETROBRAS, 2009).

No cenário ambiental, o principal organismo internacional para avaliação das mudanças climáticas é Intergovernmental Panel on Climate Change (IPCC). Utilizado como referência nacional, o órgão emite anualmente um painel apresentando os principais efeitos e observados e previsões de mudanças climáticas no mundo(UNEP, 2014).

Quando um sistema sociotécnico se desenvolve, surgem componentes que se atrasam na modernização tecnológica, chamados reversos salientes. Este conceito foi inserido na sociedade acadêmica por Hughes (1983).

O uso deste conceito é muito difundido e cresceu exponencialmente entre os anos de 1988 e 2009 (DEDEHAYIR, 2009). Entre 2009 e 2015, as citações cresceram 46\% (CAPES, 2015).

Muitos autores ao redor do mundo utilizam o conceito de reverso saliente em suas pesquisas em 
diversas áreas como Daim e outros autores (2014) em aplicações em vídeo games nos Estados Unidos, Iveroth e outros autores (2013) em sistemas urbanos na Suécia e Holanda, Dedehayir (2011) em sistemas tecnológicos na Finlândia, Taylor (2009) em Política econômica e Lehtonen (2009) em redes elétricas na Grã Bretanha.

No Brasil esse conceito ainda é pouco abordado no meio acadêmico, principalmente relacionado ao sistema energético, o que constitui uma das motivações do trabalho em questão.

O objetivo deste trabalho é analisar o sistema energético nacional como um cenário sociotécnico e evidenciar a energia como um reverso saliente de acordo com o conceito de Thomas Hughes.

\section{MÉTODO}

Com relação aos objetivos gerais, este trabalho se caracteriza como exploratório, visando proporcionar uma maior familiaridade com o problema e construir uma hipótese (GIL, 2002).

São apresentados argumentos que fundamentem a hipótese levantada. É realizada uma interpretação dos aspectos discutidos e consolidada uma conclusão do tema.

\section{RESULTADOS E DISCUSSÃO}

A produção de energia no Brasil apresenta comportamento ascendente em consonância com o crescimento populacional e desenvolvimento humano. A Figura 2 apresenta o gráfico de crescimento da produ- ção de energia no Brasil dividida pelos seus principais recursos energéticos.

Nessa Figura pode-se observar um crescimento acentuado do consumo da energia, principalmente entre os anos de 1995 e 2014. Entre os anos de 1985 e 1995 pode-se observar um período de estagnação do crescimento assim como entre 1970 e 1980 . No período entre 1980 e 1985 pode-se observar um novo intervalo de crescimento.

Figura 2 - Crescimento da produção energética no Brasil

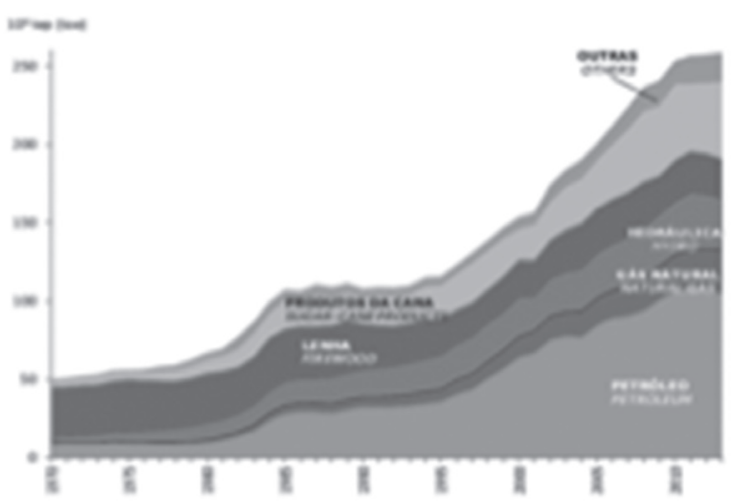

Fonte: EPE, 2014

Estas variações de comportamento intermitente da demanda energética sugerem que existem variáveis diferentes que a influenciam. Neste contexto fatores sociais, políticos, econômicos e inclusive técnicos são importantes no entendimento do sistema e reforçam a necessidade de análise do sistema energético como um sistema sociotécnico.

Aproximando-nos um pouco mais dos dias atuais, podemos observar a Figura 3. 
Figura 3 - Comportamento do consumo de energia, PIB e população no Brasil

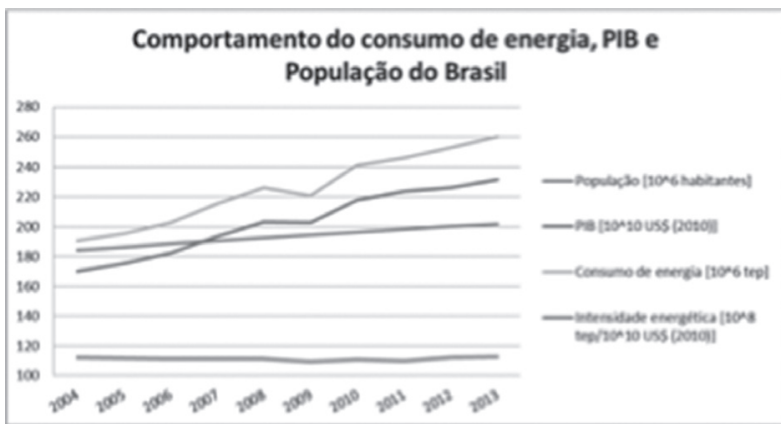

Fonte: EPE, 2014.

O Gráfico mostra que o crescimento do consumo de energia é mais elevado do que o crescimento demográfico (linha verde e azul, respectivamente). Este comportamento pode ser justificado pelo fato da ascensão das classes menos favorecidas, que passam a ter acesso à energia elétrica, e dispositivos eletrônicos (PEREIRA, 2012).

A intensidade energética, mostrada pela linha violeta do gráfico, é um indicador econômico termodinâmico e pode ser definida como a razão entre o consumo de energia e o Produto Interno Bruto (PIB) de uma economia (PATTERSON, 1996).

O consumo de energia e o PIB cresceram juntos e por isso a intensidade energética se manteve constante. Esse comportamento reforça a afirmação de Pereira (2012), já que o PIB também cresceu numa tendência mais elevada do que a população, sugerindo tendência de elevação do poder aquisitivo.

Este tipo de comportamento do consumo energético mostra a importância da abordagem sociotécnica no estudo do cenário energético nacional, pois conforme Hughes, Bijker e Pinch (1989), a mudança de comportamento de um elemento do sistema, que neste caso é a forma de consumo energético da população, faz com que os demais elementos do sistema tenham que se adaptar ao novo cenário.
Neste contexto de crescimento da demanda energética, a Figura 4 mostra a estratificação das principais fontes de energia do Brasil, mostrando que nos últimos 14 anos não se observa nenhuma mudança significativa na distribuição do consumo final.

Figura 4 - Distribuição das fontes de energia no Brasil

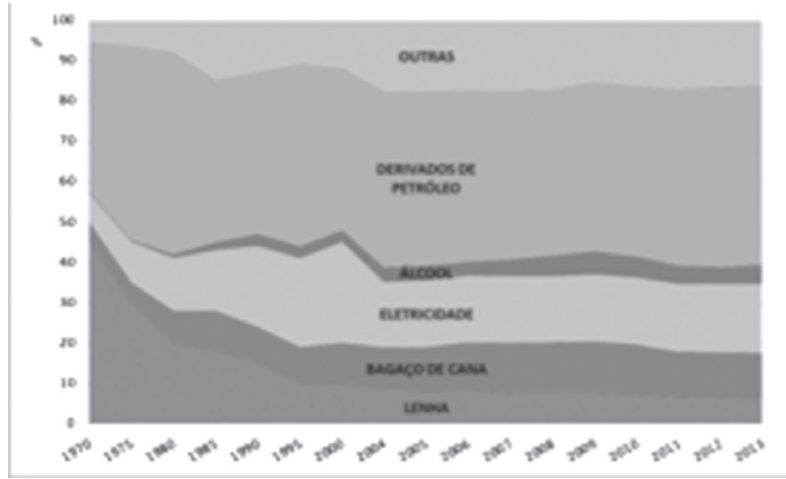

Fonte: EPE, 2014.

Com esses dados o conceito de reverso saliente energético começa a ficar mais evidente. Analisando-se os gráficos das Figuras 23 e 4 demonstra-se o crescimento da demanda de energia, a mudança nos padrões de consumo da sociedade e a estagnação da distribuição das fontes de energia nacionais, constituindo um gargalo.

Para reforçar este conceito de energia como um gargalo, baseando-se nos conceitos de Hughes (1983), podem-se analisar, também, os recursos energéticos. As Figuras 5 e 6 apresentam, respectivamente, o potencial hidrelétrico e as reservas de carvão mineral no Brasil.

Entende-se por potencial hidrelétrico o potencial possível de ser técnica e economicamente aproveitado nas condições atuais de tecnologia. O potencial hidrelétrico é medido em termos de energia firme, que é a geração máxima contínua na hipótese de repetição futura do período hidrológico crítico. 
Figura 5 - Potencial hidrelétrico brasileiro

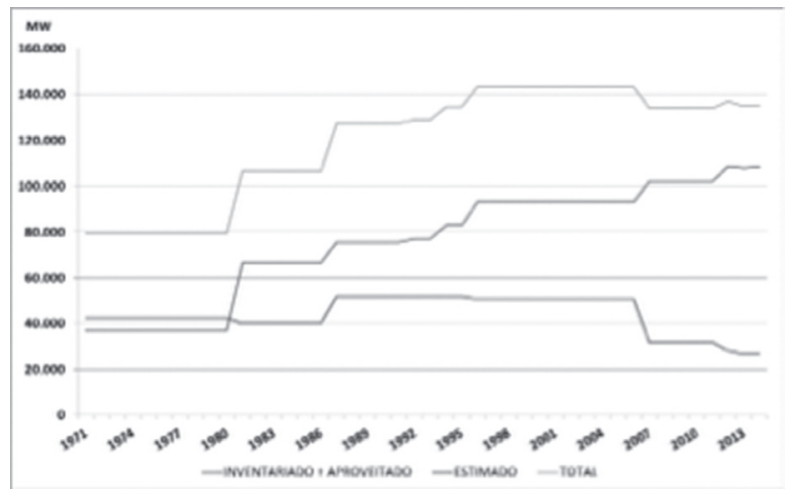

Fonte: EPE, 2014.

A energia hidráulica corresponde a $70,6 \%$ da oferta de energia elétrica nacional que está representada na Figura 4 pela faixa verde (total).

Observando-se a Figura 5 é possível notar que a partir de 1994 não houve aumento de potencial de geração de energia por usinas hidrelétricas e a partir de 2005 a tendência foi de queda. Números mais atuais apontam que de 2012 para 2013 houve redução geração em 5,9\% (EPE, 2014).

Essa questão dos reservatórios de água tem um agravante social sério. Além da redução da geração de energia elétrica, o abastecimento de água está passando por dificuldades, principalmente no estado de São Paulo. Em julho de 2014, o volume útil da Cantareira, que atende 8,8 milhões de pessoas na Grande $\mathrm{SP}$, esgotou. Com o esvaziamento do reservatório e as previsões pessimistas de falta de chuva, São Paulo se afogou na maior crise hídrica dos últimos 80 anos (COHEN, 2015).
Figura 6 - Reservas de carvão

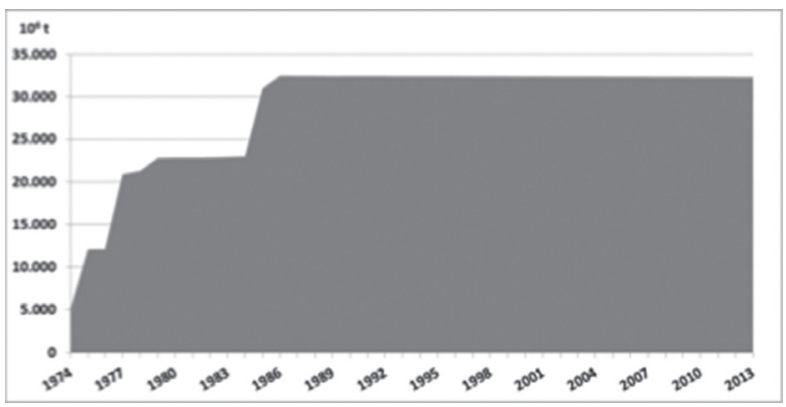

Fonte: EPE, 2014.

As reservas de carvão apresentadas no gráfico são as geológicas totais e localizadas na fonte (in situ). Para determinação das reservas recuperáveis devem ser levadas em consideração as perdas de mineração e de beneficiamento, bem como problemas de falhamento e intrusões de diabásio (EPE, 2014). A partir da Figura 6, pode-se verificar que após o ano de 1986 não foram identificadas novas reservas de carvão mineral para exploração.

Outro aspecto importante no sistema sociotécnico é o meio ambiente. A emissão total de gases do efeito estufa pelo homem no mundo aumentou em $10 \mathrm{Gt}$ de CO2 equivalente entre 2000 e 2010. Este aumento é diretamente afetado pelos setores energéticos (47\%), da indústria (30\%) e transporte (11\%).

Os principais fatores responsáveis pelo aumento destas emissões de gases com efeito de estufa antropogênico são o crescimento econômico e populacional, estilo de vida e mudanças de comportamento, mudanças associadas no uso de energia e uso da terra, tecnologia e política climática (UNEP, 2014).

Outro aspecto importante no contexto nacional são as ações governamentais. Para contornar o reverso saliente gerado o governo necessita alterar as suas estratégias de governo para buscar a estabilização do sistema sociotécnico. Como por exemplo, a criação 
em 1985 do Programa Nacional de Conservação de Energia Elétrica (Procel) pela Eletrobras.

O programa já foi responsável por poupar uma quantidade de energia elétrica que daria para abastecer miIhões de casas durante um ano inteiro. Isso foi possível por meio de ações de conscientização, contato nas escolas, nas universidades, na administração pública, participando da elaboração de novas leis e financiando projetos de combate ao desperdício. Pode-se destacar o Selo Procel, que indica aos consumidores quais são os eletrodomésticos mais eficientes. E, para incentivar a produção de eletrodomésticos cada vez mais eficientes, anualmente o Prêmio Nacional de Conservação e Uso Racional de Energia reconhece os mais econômicos em cada categoria (ELETROBRAS, 2014b).

De acordo com Felippe (2014), as ações políticas atuais têm reduzido o conjunto de desigualdades sociais. O foco tem sido o enfrentamento sem transformações na estrutura econômica, o que reduz a intervenção social à administração da miséria.

As instituições privadas, também, buscam se adaptar onde se pode ressaltar a elaboração da norma de eficiência energética ISO-50001 e a criação do Conselho Empresarial Brasileiro para o Desenvolvimento Sustentável (CEBDS).

A ISO 50001 é a norma global para a Gestão da Energia com requisitos para estabelecer uma política da energia com objetivos concretos e melhorar a eficiência energética. Definir um padrão de uso da energia, identificando as áreas críticas e compreendendo os fatores relevantes no uso de energia. Manter uma estimativa periódica do uso da energia, permitindo a visibilidade para planejar os investimentos e melhorias. Levar em consideração o consumo da energia no processo de decisão de projetos e aquisição de equipamentos, matérias-primas ou serviços (BUREAU VERITAS, 2011).

O CEBDS foi fundado em 1997 por um grupo de grandes empresários brasileiros, atento às mudan- ças e oportunidades que a sustentabilidade trazia, principalmente, a partir da Rio 92. É uma associação civil sem fins lucrativos que promove o desenvolvimento sustentável nas empresas que atuam no Brasil e hoje reúne cerca de 70 dos maiores grupos empresariais do país, que representam cerca de $40 \%$ do PIB e são responsáveis por mais de um miIhão de empregos diretos. Representante no Brasil da rede do World Business Council for Sustainable Development (WBCSD), que conta com quase 60 conselhos nacionais e regionais em 36 países, atuando em 22 setores industriais, além de contar com 200 grupos empresariais que atuam em todos os continentes (CEBDS, 2014).

\section{CONCLUSÃO}

A partir desta pesquisa foi possível evidenciar a importância da análise do sistema energético como um cenário sociotécnico, pois foram apresentadas relações complexas interdependentes entre aspectos físicos e técnicos, sociais, ambientais, econômicos e políticos, onde a cada mudança de padrão de elementos todos os demais precisam se adaptar para buscar o equilíbrio do sistema.

Foi possível identificar pontos de aderência entre os conceitos de Thomas Hughes e o sistema energético nacional, verificando o diálogo das questões de gargalo reais com os desdobramentos técnicos e sociais.

Assim, por meio da análise da tendência de crescimento da demanda de energia, a mudança nos padrões de consumo da sociedade e a estagnação da distribuição das fontes de energia nacionais apresentadas pelas figuras 2, 3 e 4, evidencia-se a produção de energia como um reverso saliente no contexto sociotécnico brasileiro e alerta-se para as necessidades de mudanças estruturais para reversão deste gargalo nacional. 


\section{REFERÊNCIAS}

ANEEL - Agência Nacional de Energia Elétrica. Relatório 2012. Brasília, 2013.

ABNT - ASSOCIAÇÃO BRASILEIRA DE NORMAS TÉCNICAS. NBR 14724: Informação e documentação - Trabalhos acadêmicos - Apresentação. Rio de Janeiro, 2011.

ABNT - ASSOCIAÇÃO BRASILEIRA DE NORMAS TÉCNICAS. NBR 6023: Informação

e documentação - Referências - Elaboração. Rio de Janeiro, 2002.

ABNT - ASSOCIAÇÃO BRASILEIRA DE NORMAS TÉCNICAS. NBR 10520: Informação e documentação - Citações em documentos - Apresentação. Rio de Janeiro, 2002.

BIJKER, W. E. \& PINCH, T. J. “The Social Construction of Facts and Artifacts: Or How the Sociology of Technology Might Benefit Each Other", Social Studies of Science, v.14, 1984. p.399-444.

BUREAU VERITAS. ISO 50001 - Sistema de Gestão em Energia. São Paulo, 2011.

CAPES. Portal de Periódicos, Brasil, 2015.

CEBDS - Conselho Empresarial Brasileiro para o Desenvolvimento Sustentável. Contribuições do Setor Empresarial Brasileiro para o Cumprimento das Metas de AICHI 2011- 2020. Rio de Janeiro, 2014.

CNI - Confederação Nacional da Indústria e ELETROBRAS. Eficiência energética na indústria: o que foi feito no Brasil, oportunidades de redução de custos e experiência internacional. Brasília, 2009.

COHEN, O. O fundo do Poço. Super Interessante, 2015. Disponível em: <http://super.abril.com.br/crise-agua/ ofundodopoco.shtml>. Acesso em: 2 abr. 2015.
DAIM, T. et al. Identifying and forecasting the reverse salient in video game consoles: A performance gap ratio comparative analysis, Technological Forecasting \& Social Change, v.82, 2014. p.177-189.

DEDEHAYIR, O.; MÄKINEN, S. J.Determining reverse salient types andevolutionary dynamics of technologysystems with performance disparities. Technology Analysis \& Strategic Management, v.23, n.10, 2011. p.1095-1114.

DEDEHAYIR, O. Bibliometric study of the reverse salient concept.Journal of Industrial Engineering and Management, Barcelona, v.2, 2009. p.569-591.

ELETROBRAS.0 Procel. Rio de Janeiro, 2014a. ELETROBRAS. Quem somos. Rio de Janeiro, 2014b.

EPE - Empresa de Pesquisa Energética. Balanço Energético Nacional 2014. Rio de Janeiro, 2014.

FELIPPE J. M. S. Os novos rumos das políticas sociais brasileiras: aproximações críticas. Interfaces Científicas - Humanas e Sociais, Aracaju, v.2, n.3, 2014. p.47-54.

GIL A. C. Como elaborar projetos de pesquisa. 4.ed. São Paulo: Atlas, 2002.

HUGHES, T. P. Networks of power: Electrification in western society, 1880-1930. The John Hopkins University Press, USA, 1983.

HUGHES, T. P.; BIJKER, W. E.; PINCH, T. J. The Social Costruction of Technological Systems. New Directions in the Sociology and History of Technology. The MIT Press, Cambridge, 1989. p. 51-82.

IVEROTH, S. P.et al. Implications of systems integration at the urban level: the case ofHammarbySjöstad. Stockholm, Journal of Cleaner Production, v.48, 2013. p.220-231. 
LEHTONEN, M.; NYE S. History of electricity network control and distribute degeneration in theUK andWesternDenmark. Energy Policy, v.37, 2009. p.2338-2345.

\section{MOON, S. SHOT Newsletter. Society for the History} of Technology, Baltimore, v.127, 2014.

PATTERSON, M. G. What is energy efficiency? Energy Police, Grã Bretanha, v.24, n.5, 1996. p.377-390.

PEREIRA, R. Consumo de energia cresce o dobro do PIB, mas fica abaixo da média mundial. Estadão. São Paulo, 2012.
RIBEIRO, A. M.; MORET, A. S. A construção da hidrelétrica de Santo Antônio e os impactos na sociedade e no ambiente. Interfaces Científicas - Humanas e Sociais, Aracaju, v.2, n.3, 2014. p.81-92.

TAYLOR, M. Z. InternationalPoliticalEconomy-The Reverse SalientofInnovationTheory. Review of Policy Research, v.26, 2009.

UNEP - UNITED NATIONS ENVIRONMENT PROGRAMME. IPCC - Intergovernmental Panel on Climate Change. Climate change 2014. Synthesis report. Genebra, Suiça, 2014.
1. Aluno de Mestrado no departamento de energia da UFABC.

Graduado em Engenharia Química pela Universidade Federal da Bahia. Trabalhando atualmente como engenheiro de processos na Unidade de Insumos básicos da Braskem no ABC paulista.

E-mail: delanomendes@hotmail.com

2. Professor Doutor na Universidade Federal do ABC no mestrado em energia, departamento de Engenharia e Modelagem. Área de eficiência energética. E-mail: sergio.lourenco@ufabc.edu.br

3. Professor Doutor na Universidade Federal do ABC no mestrado em energia, departamento de Engenharia e Modelagem. Área de eficiência energética. E-mail: douglas.cassiano@ufabc.edu.br 